\title{
THE CURRENT TEMPERATURE: A SURVEY OF POST-RESUSCITATION MANAGEMENT ACROSS AUSTRALIAN AND NEW ZEALAND INTENSIVE CARE UNITS.
}

Introduction: Post-resuscitation care has changed dramatically over the last two decades -particularly target temperature management (TTM). However, uptake across Australian and New Zealand (NZ) intensive care units (ICUs) is currently unknown.

Objectives: We aimed to describe the current status of post-resuscitation care in Australian and New Zealand ICUs.

Methods: We conducted an online survey of 162 ICU medical directors in Australia $(n=141)$ and NZ ( $n=21)$. The questionnaire consisted of multiple choice and open-ended responses.

Results: Surveys were completed for 61 ICUs (50 Australian and $11 \mathrm{NZ}$ ). The majority if ICUs were in metropolitan regions $(70 \%)$ and in were teaching hospitals (80\%), with a range of hospital bed sizes (24-875) and ICU admissions (85-3000). Two ICUs did not admit post-arrest patients (excluded from further analysis). The majority of ICUs followed a post-resuscitation care guideline (70\%). TTM was used in 57 (97\%) hospitals -but only $64 \%$ had a TTM protocol and there was widespread variation in the types of patients treated, target temperatures (range $=33-37.5 \mathrm{C}$ ), methods for cooling and duration of cooling (range=12-72 hours). TTM is commenced pre-ICU in 18\%. The majority (88\%) changed TTM practice following the 2013 TTM trial publication (100\% NZ and $85 \%$ Australia); with $28 \%$ targeting temperatures $>36 \mathrm{C}$ and $50 \%$ expressed concerns with current level of evidence for TTM. Two-thirds (31/46) of directors had reviewed the ANZCOR (Australian and New Zealand Committee on Resuscitation) TTM guideline: 16 expressed concerns with current recommendations. Less than half $(16 / 44,36 \%)$ of ICUs had a protocol for prognostication and withdrawal of treatment.

Conclusion(s): There is widespread variation in post-resuscitation care in Australian and New Zealand ICUs, and concerns with current TTM evidence and recommendations. Next, we intend to link this data to registries to examine the impact of this variation on outcomes. 